\title{
Can gravitational microlensing be used to probe geometry of a massive black-hole?
}

\author{
Luka Č. Popović and Predrag Jovanović \\ Astronomical Observatory, Volgina 7, 11160 Belgrade, Serbia \\ email: lpopovic@aob.bg.ac.yu
}

\begin{abstract}
Here we discuss the possibility to use gravitational microlensing in order to probe the geometry around a massive black hole. Taking into account that lensed quasars are emitting X-rays which come from the heart of these objects, we investigated the influence of microlensing on the Fe K $\alpha$ line shape originated in Schwarzschild and Kerr metrics.
\end{abstract}

Keywords. Accretion, accretion disks - gravitational lensing - galaxies: active

\section{Introduction}

The X-Ray emission of AGN is assumed to be generated in the innermost region of an accretion disk around a central super-massive Black Hole (BH). An emission line from iron $\mathrm{K} \alpha(\mathrm{Fe} \mathrm{K} \alpha)$ has been observed at 6-7 keV in the vast majority of AGNs (see e.g. Nandra et al. 1997). This line is probably produced in a very compact region near the $\mathrm{BH}$ and can bring essential information about the space geometry around the BH. Thus it seems clear that the $\mathrm{Fe} \mathrm{K} \alpha$ line can be strongly affected by microlensing and recent observations of three lens systems supported this idea (Popović et al. 2006).

The aim of this paper is to investigate the influence of microlensing (ML) on the AGN Fe K $\alpha$ line shape originated in a compact accretion disk around a non-rotating (Schwarzschild) and rotating (Kerr) BH and discuss the possibility that this effect can be used in probing of the massive black-hole geometry.

\section{Microlensing of an accretion disk around massive black hole}

To model an accretion disk around a massive $\mathrm{BH}$, we use the ray tracing method (Popović et al. 2003) considering only those photon trajectories which reach the sky plane at a given observer's angle $\theta_{\text {obs. }}$. If $X$ and $Y$ are the impact parameters which describe the apparent position of each point of the accretion disk image on the celestial sphere as seen by an observer at infinity, the amplified line intensity is given by

$$
I_{p}=\varepsilon(r) g^{4}(X, Y) \delta(x-g(X, Y)) A(X, Y)
$$

where $x=\nu_{\mathrm{obs}} / \nu_{0}\left(\nu_{0}\right.$ and $\nu_{\mathrm{obs}}$ are the transition and observed frequencies, respectively); $g=\nu_{\mathrm{em}} / \nu_{\mathrm{obs}}\left(\nu_{\mathrm{em}}\right.$ is the emitted frequency from the disk), and $\varepsilon(r)$ is the emissivity in the disk; $\varepsilon(r)=\varepsilon_{0} \cdot r^{-q}$. $A(X, Y)$ is the amplification caused by microlensing. To calculate $A(X, Y)$ we use three approximation for microlens: point-like, caustic and pattern microlens (see Fig. 1, and Popović et al. 2003,2006).

Here we consider an accretion disk with following parameters: $i=30^{\circ}$ and $q=2.5$, the inner radius is taken to be $R_{i n}=R_{m s}$. $\left(R_{m s}\right.$ is the radius of the marginal stability orbit; $R_{m s}=6 R_{g}$ in the Schwarzschild and $R_{m s}=1.23 R_{g}$ in the the Kerr metric with angular momentum $a=0.998$.) We adopt for the outer radius $R_{\text {out }}=20 R_{g}$. 

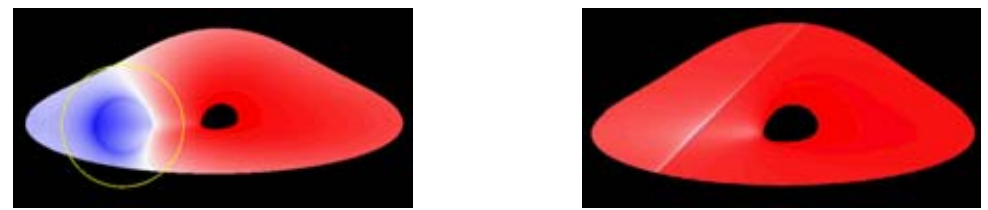

Figure 1. Simulations of the accretion disk microlensing by point-like microlens (in the Kerr metric, left) and straight-fold caustic (in the Schwarzschild metric, right).
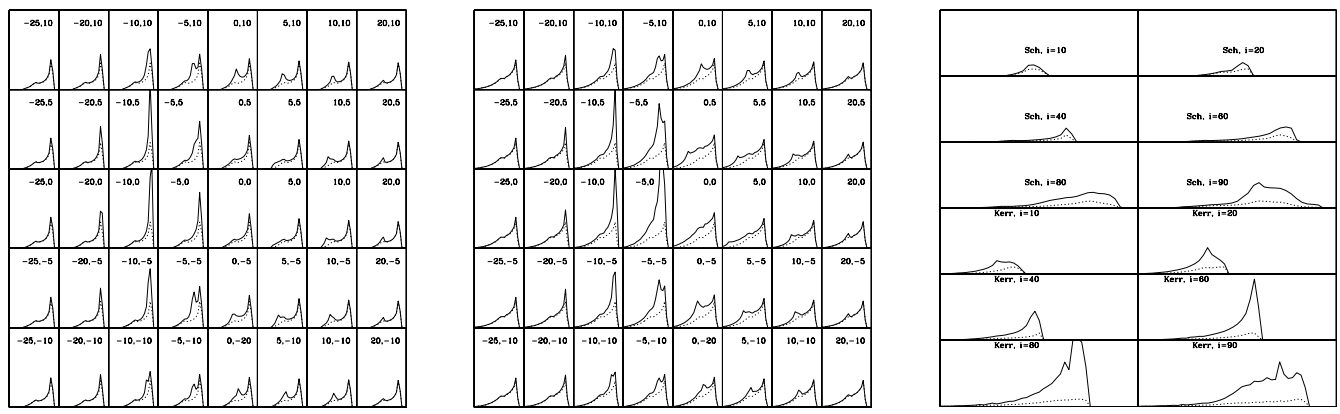

Figure 2. The amplification and deformation of the $\mathrm{Fe} \mathrm{K} \alpha$ line in the case of Schwarzschild (left panel) and Kerr (middle panel) metrics. The different shapes and amplification of the Fe $\mathrm{K} \alpha$ line for different inclinations (right panel).

Some of our results are presented in Fig. 2 (also see Popović et al. 2003, 2006) where the difference of the $\mathrm{Fe} \mathrm{K} \alpha$ line amplification in Schwarzschild (first panel) and Kerr (second panel) metrics are given for different positions of the point-like lens $\left(\mathrm{X}, \mathrm{Y}\right.$ in $\left.R_{g}\right)$ across the accretion disk (first two panels). Third panel of Fig. 2 shows the shape of Fe K $\alpha$ line (dashed line) for different inclination and its deformation induced by ML (solid line) for a point-like microlens of $\mathrm{EER}=10 \mathrm{R}_{g}$, located at position of $X_{0}=-5 R_{g}, Y_{0}=5 R_{g}$ in both metrics; Schwarzschild (Sch) and Kerr (Kerr).

Several outstanding changes of the line shape with the location of the microlens, and consequently with the transit of a microlens across the disk, can be inferred from Fig. 2 in both metrics: (i) the number of peaks, their relative separation and the peak velocity could change (this also affects to the velocity centroid), (ii) an asymmetrical enhancement of the line profile, (iii) amplification has a maximum for negative values of $X_{0}$ that correspond to the approaching part of the rotating disk.

On the other hand, as one can see from Fig. 2, the amplification and deformation of the Fe $\mathrm{K} \alpha$ line originated in the Kerr metric are more prominent that in the Schwarzschild one. Detailed discussion of our investigations can be found in Popović et al. $(2003,2006)$ and Jovanović (2006). The obtained results show that microlensing can be used in investigations of an unresolved X-ray emitting region geometry around massive black holes.

\section{Acknowledgements}

This work was supported by the Ministry of Science and Environment Protection of Serbia through the project 146002 .

\section{References}

Jovanović, P. 2006, PASP, 118, 656

Nandra, K., George, I. M., Mushotzky, R. F., Turner, T. J. \& Yaqoob, T. 1997, ApJ, 477, 602

Popović, L. Č., Jovanović, P., Mediavilla, E. et al. 2006, ApJ, 637, 620

Popović, L. Č., Mediavilla, E. G., Jovanović, P. \& Muñoz, J. A. 2003, A\&A, 398, 975 